\title{
Self-rated general and oral health and associated factors in independently-living older individuals
}

\author{
Luísa Helena do Nascimento \\ TÔRRES(a) iD \\ Maria Laura Braccini \\ FAGUNDES $^{(a)}$ iD \\ Débora Dias da SILVA ${ }^{(b)}$ \\ Anita Liberalesso NERI(c) \\ Juliana Balbinot HILGERT(d) iD \\ Fernando Neves HUGO(d) iD \\ Maria da Luz Rosário de SOUSA(e) \\ (a) Universidade Federal de Santa Maria \\ - UFSM, Dental School, Department of \\ Stomatology, Santa Maria, RS, Brazil. \\ (b) Universidade Paulista - UNIP, Dental \\ School, Departament of Community and \\ Dental Health, Campinas, SP, Brazil. \\ (c) Universidade Estadual de Campinas - \\ Unicamp, Faculty of Medical Sciences, \\ Departament of Genrontology, Campinas, \\ SP, Brazil. \\ (d) Universidade Federal do Rio Grande do \\ Sul - UFRGS, Dental School, Departament \\ of Preventive and Social Dentistry, Porto \\ Alegre, RS, Brazil. \\ (e) Universidade Estadual de Campinas - \\ Unicamp, Dental School, Department of \\ Health Sciences and Pediatric Dentistry, \\ Piracicaba, SP, Brazil.
}

Declaration of Interests: The authors certify that they have no commercial or associative interest that represents a conflict of interest in connection with the manuscript.

Corresponding Author:

Luísa Helena do Nascimento Tôrres

E-mail: Ihntorres@gmail.com

htips://doi.org/10.1590/1807-3107bor-2020.vol34.0079

Submitted: September 3, 2019

Accepted for publication: April 20,2020

Last revision: May 22, 2020
Abstract: The aim of this study was to verify the association between sociodemographic, general health, and oral health data with self-rated general health (SRGH) and self-rated oral health (SROH) in independent-living older Brazilians. This cross-sectional study was part of a larger study with older individuals living independently in the city of Campinas, Brazil - the "Rede FIBRA" Study (the Frailty in Brazilian Elderly Study). A random sample of 688 older individuals responded the SRGH and 673, the SROH. SRHG and SROH were both assessed using a single item. The questionnaire included sociodemographic, general, and oral health data. The mean age was $72.28 \pm 5.4$ years. The adjusted analysis revealed that the probability of rating general health as bad was higher for illiterate participants (PR: 1.77, 95\%CI: 1.13-2.77) or with low educational level (PR: 1.76, 95\%CI: 1.17-2.65), those with depressive symptoms (PR: 1.45, 95\%CI:1.21-1.74), participants that self-reported food limitation due to problems with denture or lack of it (PR: 1.29, 95\%CI: 1.05-1.56), and those with xerostomia (PR 1.40, 95\%CI: 1.17-1.67). The probability of rating general health as bad was lower for participants that presented 0-2 chronic diseases (PR: 0.64, 95\%CI: 0.53-0.78) and were pre-frail (PR: 0.76, 95\%CI: 0.61-0.96). With regard to $\mathrm{SROH}$, the probability of rating oral health as bad was higher for participants with natural teeth (PR:1.61, 95\%CI: 1.24-2.08), that reported xerostomia (PR: 1.44, 95\%CI: 1.13-1.84), and food limitation due to problems with denture or lack of it (PR: 1.43, 95\%CI: 1.07-1,91), and lower for participants that reported having enough money to cover daily expenses (PR: 0.78, 95\%CI: 0.61-0.99). Oral health data and income seem to be related to self-perceptions of general and oral health.

Keywords: Aged; Oral Health; Self Concept; Health Services for the Aged.

\section{Introduction}

With population aging, the prevalence of chronic diseases and limitations due to physiological decline and use of medications increase. Therefore, it is important to understand how older people deal with age-related changes. The clinical assessment of health status may underestimate the adaptive strategies for coping with difficulties and limitations. For the elderly, an individual evaluation of health is considerably influenced by the level of emotional well-being and physical function. ${ }^{1}$ Previous studies 
have indicated that psychosocial factors, such as somatic symptoms, hypochondriacal attitudes, and social and mental well-being, explained the variance in self-rated health to a greater extent compared to physical health facors. ${ }^{2}$ Moreover, subjective health optimism appears to play a protective role, ${ }^{3}$ as shown in a study in which resilience appeared to be a protective mechanism against the deleterious effects of tooth loss. ${ }^{4}$

Lack of communication between patient and physician/dentist might affect their relationship, impair treatment, and contribute to health decline. Several studies in different areas have assessed the disagreement between a patient's perceptions and those of the family, caregivers, or the physician's diagnosis, representing different views of the same situation. ${ }^{2}$ Particular attention must be given to elders, because they may be influenced by their own choices and decisions.

Self-rated health status is one of the most used health indicators, ${ }^{5}$ and health perception seems to be an important predictor of mortality. ${ }^{6}$ Poorer selfrated health (SRH) is associated with poorer health outcomes, greater disease severity, and symptom burden. ${ }^{7}$ Poor health status of mouth and teeth can restrict function and cause discomfort, and, as all body parts, also affect the self-rated general health. ${ }^{8,9}$ Due to common risk indicators, such as poor nutrition caused by tooth loss, oral health and general health are interrelated especially among older individuals.

Previous epidemiological studies have shown associations between self-ratings and well-being, somatic conditions, and body mass index., ${ }^{1,2}$ Hence, assessing general health and oral health self-ratings can contribute to a joint action among different professionals. Oral health perception is an important indicator because it summarizes objective health conditions, subjective responses, and values and cultural expectations. ${ }^{10}$ Furthermore, the relationship between oral health and general health is complex and multifaceted, especially in the elderly, ${ }_{11}^{11}$ in whom general and oral health are more interconnected. According to the authors, some general illnesses that are more prevalent among the elderly may act as predisposing factors for oral health disability, as for example, diabetes that can lead to xerostomia. On the other hand, common oral diseases such as tooth loss and periodontitis may act as predisposing factors for malnutrition and may restrict the consumption of certain foods.

Therefore the aim of this study was to verify the association between sociodemographic, general health, and oral health data with self-rated general and oral health in independent-living older Brazilians. It was hypothesized that SRGH and SROH would have common risk indicators.

\section{Methods}

\section{Study design}

This cross-sectional study was part of a larger study on independent-living older individuals from the city of Campinas, Brazil - the "Rede FIBRA" study (Frailty in Brazilian Elderly Study), which is a multicenter and multidisciplinary study aimed at identifying conditions of frailty in the elderly ( $\geq 65$ years) from the community to better understand the prevalence, characteristics, and main factors associated with frailty in Brazilian elderly population.

\section{Sample size}

Additional details about data collection procedures were published elsewhere. ${ }^{12}$ The cognitive status of the participants was assessed with the MMSE (Mini-Mental State Examination) and independence was assessed by their capacity to go to the location where data was collected. If participants did not reach the cut-off point for $\mathrm{MMSE}^{13}$ according to their schooling (less one standard deviation) they were excused from the second part of the protocol because cognitive deficits could undermine the reliability of self-reported responses. The inclusion criteria were: 65 years old or more, being able to understand the instructions, living permanently in their home (i.e. in the community and in their houses) and being in the census tract. The exclusion criteria comprised severe cognitive impairment, temporary or permanent inability to walk (the use of cane and walker were allowed), localized strength loss and aphasia due to serious stroke, serious impairment due to Parkinson, severe communication difficulties, chemotherapy treatment, severe sensory deficit, and being in the terminal stage of a disease. 
Ninety participants were randomly selected from the 835 urban census tracts (about 10 per tract) of Campinas according FIBRA Study criteria. The estimated sample size was of 601 older people living independently in the community (Figure). The sample size was overestimated by $50 \%$ in order to account for refusals and exclusions, resulting in 900 participants. ${ }^{11}$ The minimum sample size was calculated by the finite population formula to obtain statistical significance for describing the prevalence of biological frailty, use and need of dental prosthesis, presence of natural teeth and soft tissue alterations with prevalence estimates of $50 \%$ each (maximum margin of safety) and sampling error of $4 \%$ for the general purpose of the FIBRA study. ${ }^{11}$ After completing the first part of the protocol, the MMSE was applied and, if the score was lower than the cut-off point, the participant was considered not eligible $(n=211)$ since cognitive issues could interfere with the ability to answer the questions that were part of the study protocol. If MMSE scores were above the cut-off point, the participant answered the second part of the protocol, which included the self-report of physical health conditions, functionality, expectation of care, depressive symptoms, and life satisfaction. ${ }^{12}$

Of the 900 volunteers, 689 participated in the complete protocol and of those 688 completed the protocol for SRGH and 673 for SROH (i.e. older persons for whom questionnaire information on these data were available) and were analyzed in the present study.

\section{Ethics}

This study was conducted after approval from the Ethics Committee on Research involving Human Beings of the Piracicaba Dental School/University of Campinas ( $\mathrm{N}^{\circ}$. 15/2009). The purpose of the study was explained to participants before they were asked to sign the informed consent. The interview started with identification and MMSE application.

\section{Interview and data collection}

\section{Outcomes}

Self-rated general health and oral health were both assessed using a single item. Participants were asked to rate their general health and oral health based on

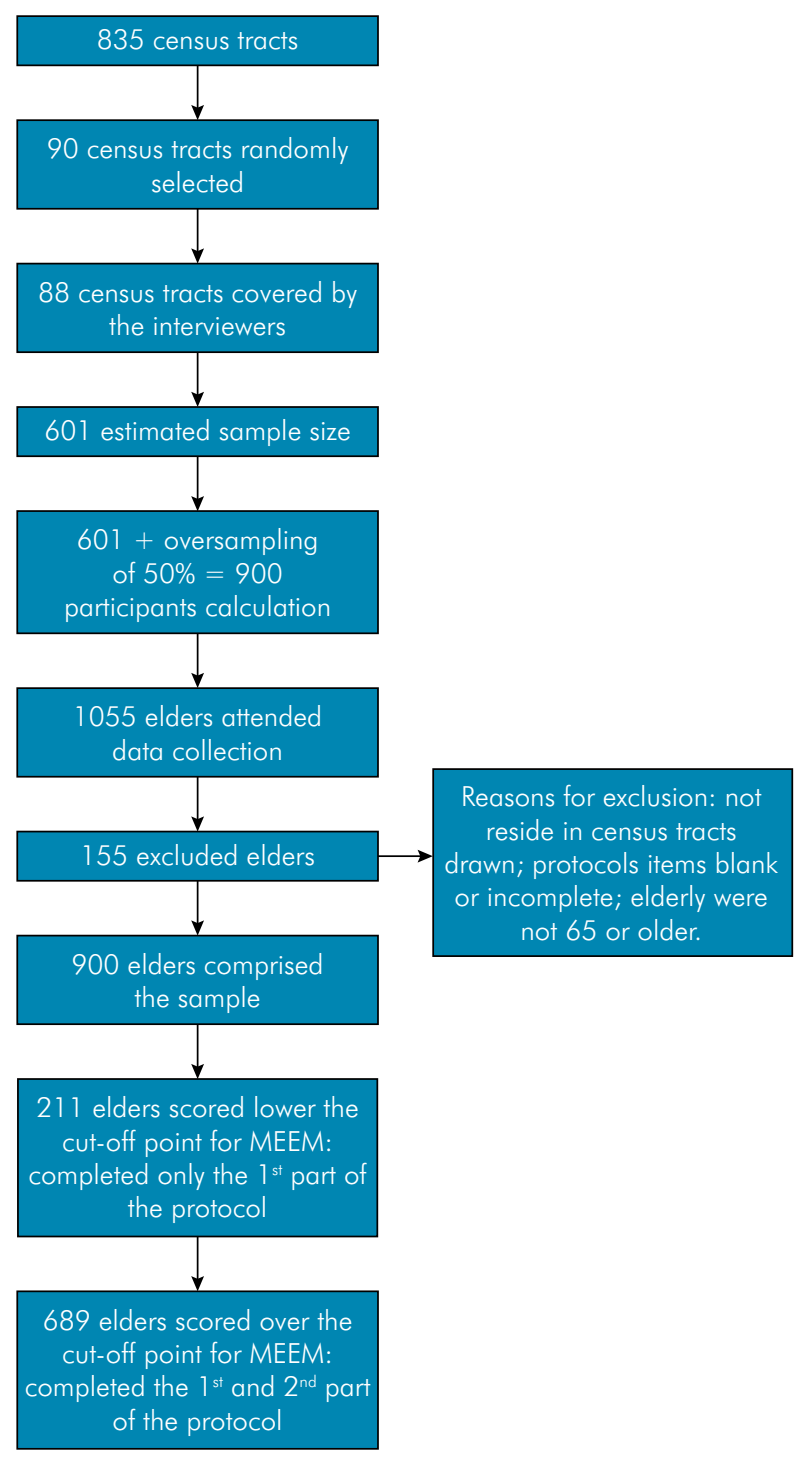

Figure. Flowchart of the sampling procedure.

the questions: "How do you rate your general health?" and "How do you rate your oral health?". For both questions the possible answers were: very good, good, regular, bad, or very bad. These were afterwards grouped into two categories: good (good/very good) and bad (regular/bad/very bad).

\section{Sociodemographic data and smoking status}

Sociodemographic data included age (in years), self-reported socio-economic status [assessed trough the question "Do you think you (and your partner) have enough money to cover daily expenses?", yes or no), schooling (in years), self-reported race/skin 
color (white/ black/ brown/ indigenous/asian), sex (male/female), and marital status (married/ single/ divorced/ widow). Sociodemographic variables and current smoking status (assessed through the question "Do you currently smoke?" (yes/no) were assessed in an interview to answer the questionnaire. Age and schooling were transformed from continuous into ordinal variables (age-group 65-69/ 70-74/ 75-79/ $\geq 80$ years-old and illiterate/ $1-8$ years/ $>8$ years, respectively). Race/skin color and marital status were categorized into white/non-white and married/ widow, single, divorced.

\section{General health assessment}

The variable "number of chronic diseases" (heart problem/ hypertension/ stroke/ diabetes mellitus/ cancer/ arthritis/ lung disease/ depression/ osteoporosis) was created based on the self reported diseases. A summary measure was used in the analyses.

Depressive symptoms were assessed with the Geriatric Depression Scale (GDS) - Brazilian Portuguese short version ${ }^{14}$. The cut-off point was $\leq 5$ for absence and $>5$ for presence of symptoms.

The frailty criterion as proposed by Fried et a ${ }^{15}$ is determined by the presence of three or more of the following criteria: unintentional weight loss, self-reported exhaustion, weakness (decreased grip strength), slow walking speed, and low physical activity level. Participants were categorized into non-frail ( 0 criteria), pre-frail ( 1 or 2 criteria), or frail ( 3 or more).

\section{Oral health assessment}

Oral health was assessed by self-reported food limitation, xerostomia, presence or absence of natural teeth, and denture use. They were each measured by single questions:

a. Self-reported food limitation was assessed with the question "How often did you limit the types or amount of food you eat because of problems with your teeth or dentures (in the past three months)?" with always, sometimes and never as possible answers. Afterwards, always and sometimes were combined and considered as yes;

b. Presence and absence of natural teeth was assessed with the question "Do you have any natural teeth?" (yes or no); c. Denture use was assessed by the question "Do you wear a denture?" (yes or no);

d. Xerostomia was assessed by the question "Have you experienced dry mouth in the last 4 weeks?" (yes or no).

Associations with the outcomes were adjusted for sociodemographic variables, including age (65-69/70-74/75-79/ $\geq 80$ years), race/skin color (white/non-white), sex (female/male), marital status (married/ widow, single, divorced), schooling (illiterate/1-8 years/ $>8$ years), and having enough money to cover daily expenses (yes/no). Associations were also adjusted for depression symptoms (present/absent), smoking status (yes/no), frailty (not frail / pre-frail/frail), number of chronic diseases $(0-2 / \geq 3$ diseases), and oral status represented by the presence of natural teeth (present/ absent), denture use (yes/ no), xerostomia (yes/ no), and self-reported food limitation due to problems with denture or lack of it (yes/ no).

The variables that presented two possible answers (i.e. variables that were dichotomized) were coded as 0 and 1, being 1 always given for the worst condition. The variables that had three possible answers (as schooling and frailty) were coded in an ascending order, from best to worst.

\section{Statistical analyses}

Two separate analyses were performed for each outcome using the same procedures. The outcomes were dichotomized into good and bad self-perception. Categorical data are presented as counts and percentage. Chi-square test was performed to assess differences between the studied variables including sociodemographic, behavioral, and general health and oral health assessments with the outcomes. To test the study hypothesis, all independent variables that showed association with $p<0.25$ in the bivariate analyses were candidates for the multivariate Poisson regression model. Also, all epidemiological relevant variables were included in the analysis. Variables that did not contribute to the model ( $p>0.25$ ) were excluded and a new model was developed. The old and new models were always compared. The model was developed using the Enter (backwards) method. A Poisson regression 
analysis with robust variance was used to estimate crude and adjusted prevalence ratio (PR), and 95\% confidence intervals $(95 \% \mathrm{CI})$ were calculated for the variables of interest with both the outcomes (SRGH and $\mathrm{SROH}$ ). Unadjusted Poisson analyses were performed to provide a preliminary assessment of the association between covariates and the outcomes; the adjusted regressions were used to evaluate the final associations, verifying if the covariates were common to both outcomes. All statistical analyses were carried out using the PASW Statistics software version 18 (IBM SPSS Statistics 18, NY).

\section{Results}

The characteristics of the study participants are presented in Table 1. Of the 689 participants (mean age $72.28( \pm 5.4)$ years $), 47 \%$ had edentulism $(n=315)$ and $16 \%$ had 20 or more teeth. The majority was women (68.3\%) and white (73.4\%). A hundred and eighty four (27.6\%) reported bad oral health (very bad - 0/ bad $3.8 \%$ / regular $23.8 \%$ ) and $283(41.1 \%)$ reported bad general health (very bad $-1.3 \%$ / bad $4.5 \%$ / regular $35.3 \%$ ). Of those reporting bad general health, 15\% also reported bad oral health and $45.9 \%$ (306) reported good general health and good oral health $(\mathrm{p}<0.001$, data not shown). In the total sample, $8 \%$ were frail.

The adjusted analysis revealed that the probability of rating general health as bad was higher for illiterate participants (PR: 1.77, 95\%CI: 1.13-2.77) or with low educational level (PR: 1.76, 95\%CI: 1.17-2.65), with depressive symptoms (PR: 1.45, 95\%CI: 1.21-1.74), that self-reported food limitation due to problems with denture or lack of it (PR: 1.29, 95\%CI: 1.05-1.56), and xerostomia (PR: 1.40, 95\%CI: 1.17-1.67). The probability of rating general health as bad was lower for participants with 0-2 chronic diseases (PR: 0.64, 95\%CI: 0.53-0.78) and who were pre-frail (PR: 0.76, 95\%CI: 0.61-0.96) compared to frail participants.

With regard to self-rated oral health, in the adjusted analysis the probability of rating oral health as bad was higher for participants with natural teeth (PR: 1.61, 95\%CI: 1.24-2.08), that reported xerostomia (PR: 1.44, 95\%CI: 1.13-1.84) and food limitation (PR: 1.43, 95\%CI: 1.07-1.91), and lower for participants that reported having enough money to cover daily life needs (PR: 0.78, 95\%CI: 0.61-0.99) - Table 2. The fit of each model was assessed using AIC (Akaike Information Criteria, lower values indicating better fit) and BIC (Bayesian Information Criteria, lower values indicating better fit).

Both models were tested, and overdispersion was not observed (data not shown).

\section{Discussion}

In the present study, we sought to evaluate health and oral health by physical, behavioral, and mental health characteristics in a group of elderly persons. The same variables were used to assess the factors related to SRGH and SROH. Our results suggest that there are common explanatory variables related to both outcomes, since two out of three oral health variables were associated with both SRGH and $\mathrm{SROH}$. According to Okunseri et al., ${ }^{16}$ who studied an ethnic minority group, participants who reported good/excellent oral health were almost six times more likely to report good/excellent general health. In another study with residents of a retirement community, $\mathrm{SROH}$ alone added $5.4 \%$ to the variance explained in SRGH showing that $\mathrm{SROH}$ is related to people's perceptions of themselves, which is an important aspect when rating their health and their ability to function well ${ }^{8}$. In addition, in the same way as general health, oral health may symbolize old age and fears of limited function, dependence, poor appearance, discomfort, and pain. ${ }^{8}$

Xerostomia was associated with higher prevalence of both bad SRGH and SROH. This condition is a quite common complaint in the elderly population, ${ }^{17}$ possibly because of polypharmacy and discomfort affecting their quality of life. Matear et al. ${ }^{17}$ observed a relationship between xerostomia and poor general health, and associated this with the presence of diseases and respective medications. Further, self-report of food limitation was also associated with both SRGH and SROH. This might reflect limited physical function and is probably the reason why both self-perceptions were affected in older participants. According to Inukai et al., ${ }^{23}$ difficulty with chewing can have a direct or indirect impact on the psychological and social dimensions of oral 
Self-rated general and oral health and associated factors in independently-living older individuals

Table 1. Socio-demographic, personal, general, and oral health characteristics and frequency distributions of study sample according to the outcomes SRGH $(n=688)$ and SROH $(n=673)$.

\begin{tabular}{|c|c|c|c|c|}
\hline \multirow{2}{*}{ Variables } & Good SRGH* & Bad SRGH* & Good SROH** & Bad SROH** \\
\hline & $\mathrm{n}(\%)$ & $\mathrm{n}(\%)$ & $\mathrm{n}(\%)$ & $\mathrm{n}(\%)$ \\
\hline \multicolumn{5}{|l|}{ Sex } \\
\hline Female & $272(67.2 \%)$ & $198(70.0 \%)$ & $341(70.0 \%)$ & $124(66.7 \%)$ \\
\hline \multicolumn{5}{|c|}{ Self-report of enough money to cover daily expenses } \\
\hline Yes & $251(62.8 \%)$ & $145(51.4 \%)$ & $292(60.6 \%)$ & $97(52.4 \%)$ \\
\hline \multicolumn{5}{|l|}{ Schooling } \\
\hline Illiterate & $57(14.1 \%)$ & 56 (19.8\%) & $77(15.8 \%)$ & $34(18.3 \%)$ \\
\hline $1-8$ years & $263(64.9 \%)$ & $207(73.1 \%)$ & $336(69.0 \%)$ & $124(66.7 \%)$ \\
\hline$>8$ years & $85(21.0 \%)$ & $20(7.1 \%)$ & $74(15.2 \%)$ & $28(15.1 \%)$ \\
\hline \multicolumn{5}{|l|}{ Race/skin color } \\
\hline White & $313(77.3 \%)$ & $192(67.8 \%)$ & $366(75.2 \%)$ & 129 (69.4\%) \\
\hline \multicolumn{5}{|l|}{ Marital status } \\
\hline Married & $226(55.8 \%)$ & $151(53.4 \%)$ & $266(54.6 \%)$ & $102(54.8 \%)$ \\
\hline \multicolumn{5}{|l|}{ Age-group } \\
\hline 65-69 years-old & $148(36.5 \%)$ & 102 (36.0\%) & 170 (34.9\%) & $75(40.3 \%)$ \\
\hline 70-74 years-old & 125 (30.9\%) & $102(36.0 \%)$ & $164(33.7 \%)$ & $61(32.8 \%)$ \\
\hline $75-79$ years-old & 83 (20.5\%) & 53 (18.7\%) & 99 (20.3\%) & $33(17.7 \%)$ \\
\hline$\geq 80$ years-old & $49(12.1 \%)$ & $26(9.2 \%)$ & $54(11.1 \%)$ & $17(9.1 \%)$ \\
\hline \multicolumn{5}{|c|}{ Number of chronic diseases } \\
\hline 0-2 diseases & $276(68.1 \%)$ & $115(40.8 \%)$ & $286(59.1 \%)$ & $93(50.5 \%)$ \\
\hline \multicolumn{5}{|l|}{ Depressive symptoms } \\
\hline Present & $44(11.1 \%)$ & $88(31.8 \%)$ & 87 (18.0\%) & $44(24.2 \%)$ \\
\hline \multicolumn{5}{|l|}{ Frailty } \\
\hline Not frail & $191(47.2 \%)$ & $112(39.6 \%)$ & $223(45.8 \%)$ & $74(39.8 \%)$ \\
\hline Pre-frail & 204 (50.4\%) & 147 (51.9\%) & $241(49.5 \%)$ & 99 (53.2\%) \\
\hline Frail & $10(2.5 \%)$ & $24(8.5 \%)$ & $23(4.7 \%)$ & $13(7.0 \%)$ \\
\hline \multicolumn{5}{|l|}{ Smoking status } \\
\hline Yes & 45 (11.1\%) & $32(11.3 \%)$ & 49 (10.1\%) & $25(13.6 \%)$ \\
\hline \multicolumn{5}{|l|}{ Natural teeth } \\
\hline Present & $225(57.3 \%)$ & $131(47.0 \%)$ & $239(49.3 \%)$ & $116(62.7 \%)$ \\
\hline \multicolumn{5}{|c|}{ Self-reported food limitation due to problems with denture or lack of it } \\
\hline Yes & $40(10.2 \%)$ & $55(19.9 \%)$ & $56(11.9 \%)$ & $35(19.1 \%)$ \\
\hline \multicolumn{5}{|l|}{ Denture use } \\
\hline Yes & $253(67.3 \%)$ & $205(75.1 \%)$ & $342(72.6 \%)$ & $116(64.8 \%)$ \\
\hline \multicolumn{5}{|l|}{ Xerostomia } \\
\hline Yes & $144(36.5 \%)$ & $152(55.1 \%)$ & 199 (40.9\%) & $101(54.3 \%)$ \\
\hline
\end{tabular}

*Self-rated general health; ${ }^{* *}$ Self-rated oral health.

health. The association of these two covariates with the outcomes may be interpreted as weak, since there are other factors such as age itself that are extremely relevant for perceived health and perceived oral health status.

The absence of teeth may affect food choice and nutritional status, and thus influence general health condition. Interestingly, although tooth loss and denture use are considered natural characteristics of the aging process among the elderly, denture use was not associated with any of the outcomes, but the presence of natural teeth was associated with higher prevalence of bad $\mathrm{SROH}$. A possible explanation for this could be related to the identification of other 
Table 2. Crude and adjusted prevalence ratios and $95 \%$ confidence intervals $(\mathrm{CI})$ of the variables associated with SRGH and SROH.

\begin{tabular}{|c|c|c|c|c|}
\hline \multirow{3}{*}{ Variables } & \multicolumn{2}{|c|}{$\mathrm{SRGH}^{*}$} & \multicolumn{2}{|c|}{$\mathrm{SROH}^{* *}$} \\
\hline & Crude & Adjusted & Crude & Adjusted \\
\hline & PR $(95 \% \mathrm{Cl})$ & PR $(95 \% \mathrm{Cl})$ & PR $(95 \% \mathrm{Cl})$ & PR $(95 \% \mathrm{Cl})$ \\
\hline \multicolumn{5}{|l|}{ Sex } \\
\hline Female & $\begin{array}{c}1.08 \\
(0.88-1.31)\end{array}$ & $\begin{array}{c}0.93 \\
(0.77-1.13)\end{array}$ & - & - \\
\hline Male & 1 & 1 & & \\
\hline \multicolumn{5}{|c|}{ Self-report of enough money to cover daily expenses } \\
\hline Yes & $\begin{array}{c}0.76 \\
(0.64-0.91)\end{array}$ & $\begin{array}{c}0.91 \\
(0.77-1.09)\end{array}$ & $\begin{array}{c}0.75 \\
(0.58-0.98)\end{array}$ & $\begin{array}{c}0.78^{\circ} \\
(0.61-0.99)\end{array}$ \\
\hline No & 1 & 1 & 1 & 1 \\
\hline \multicolumn{5}{|l|}{ Schooling } \\
\hline Illiterate & $\begin{array}{c}2.60 \\
(1.68-4.02)\end{array}$ & $\begin{array}{c}1.77^{a} \\
(1.13-2.77)\end{array}$ & - & - \\
\hline $1-8$ years & $\begin{array}{c}2.31 \\
(1.53-3.47)\end{array}$ & $\begin{array}{c}1.76^{\mathrm{b}} \\
(1.17-2.65)\end{array}$ & - & - \\
\hline$>8$ years & 1 & 1 & & \\
\hline \multicolumn{5}{|c|}{ Number of chronic diseases } \\
\hline 0-2 diseases & $\begin{array}{c}0.52 \\
(0.43-0.62)\end{array}$ & $\begin{array}{c}0.64^{\mathrm{b}} \\
(0.53-0.78)\end{array}$ & - & - \\
\hline$\geq 3$ diseases & 1 & 1 & & \\
\hline \multicolumn{5}{|c|}{ Depressive symptoms } \\
\hline Present & $\begin{array}{c}1.91 \\
(1.62-2.26)\end{array}$ & $\begin{array}{c}1.45^{\mathrm{b}} \\
(1.21-1.74)\end{array}$ & - & - \\
\hline Absent & 1 & 1 & & \\
\hline \multicolumn{5}{|l|}{ Frailty } \\
\hline Not frail & $\begin{array}{c}0.52 \\
(0.40-0.68)\end{array}$ & $\begin{array}{c}0.81 \\
(0.62-1.04)\end{array}$ & - & - \\
\hline Pre-frail & $\begin{array}{c}0.59 \\
(0.46-0.76)\end{array}$ & $\begin{array}{c}0.76^{a} \\
(0.61-0.96)\end{array}$ & - & - \\
\hline Frail & 1 & 1 & & \\
\hline \multicolumn{5}{|l|}{ Natural teeth } \\
\hline Present & $\begin{array}{c}0.78 \\
(0.65-0.94)\end{array}$ & $\begin{array}{c}0.85 \\
(0.72-1.01)\end{array}$ & $\begin{array}{c}1.45 \\
(1.10-1.92)\end{array}$ & $\begin{array}{c}1.61^{\mathrm{b}} \\
(1.24-2.08)\end{array}$ \\
\hline Absent & 1 & 1 & 1 & 1 \\
\hline \multicolumn{5}{|c|}{ Self-reported food limitation due to problems with denture or lack of it } \\
\hline Yes & $\begin{array}{c}1.50 \\
(1.23-1.84)\end{array}$ & $\begin{array}{c}1.29^{a} \\
(1.05-1.56)\end{array}$ & $\begin{array}{c}1.54 \\
(1.14-2.07)\end{array}$ & $\begin{array}{c}1.43^{\circ} \\
(1.07-1.91)\end{array}$ \\
\hline No & 1 & 1 & 1 & 1 \\
\hline \multicolumn{5}{|l|}{ Xerostomia } \\
\hline Yes & $\begin{array}{c}1.55 \\
(1.29-1.86)\end{array}$ & $\begin{array}{c}1.40^{\mathrm{b}} \\
(1.17-1.67)\end{array}$ & $\begin{array}{c}1.53 \\
(1.18-1.99)\end{array}$ & $\begin{array}{c}1.44^{\mathrm{b}} \\
(1.13-1.84)\end{array}$ \\
\hline No & 1 & 1 & 1 & 1 \\
\hline
\end{tabular}

${ }^{a} p<0.05 ;{ }^{b} p<0.01 ; *$ Self-rated general health; ${ }^{* *}$ Self-rated oral health.

factors that may be considered more important to older individuals than having teeth. Moreover, the results suggest that perceptions are more carefully assessed in $\mathrm{SROH}$, so the expectation and evaluation 
of a person's natural teeth condition is more rigorous, leading older participants to consider this aspect in their critical judgment. In addition, it is possible that the self-report of having natural teeth might be a good estimator of oral clinical status but may lack ability to identify self-perception of general health. Another possibility is that the self-perception of general health is not affected by the number of natural teeth whereas for some elders tooth extraction can 'prevent' future pain..$^{18}$ Gibson et al. ${ }^{19}$ assessed homeless veterans perception and those who received emergency dental care showed a decline in their GOHAI scores (General Oral Health Assessment Index) from baseline until after dental treatment, in comparison with those who received comprehensive dental care. These findings suggest that the use of health services may contribute to the sense of awareness that amplifies perceptions. ${ }^{20,21}$ Moreover, when comparing preventive dental care and restorative work, those who visited the dentist for regular check-ups were more likely to report their oral health to be good/excellent. ${ }^{16}$ According to Haikal et al.,22 who assessed the perception of elderly persons living in a Brazilian facility, those who rated their oral health as poor or fair reported the need to visit the dentist, suggesting that self-perception of oral health is a predictor of need for dental care. However, teeth esthetics, position, and functional status were not analyzed, and this may have resulted in a study limitation.

Having no or few diseases was related to a lower perception of bad general health. Although Kieffer and Hoogstraten ${ }^{24}$ studied young adults, they also found that those who reported more health symptoms seemed to regard their general health as being worse. A previous longitudinal research with older adults revealed that those who experienced fewer health problems and functional impairment felt more in control later in life. ${ }^{6}$ The aggregation of chronic diseases into two categories (0-2 / $\geq 3$ diseases) was done based on studies that examined the association of comorbidities and perceived health. ${ }^{25}$

Mental health changes have been closely related to changes in self-rated health. ${ }^{7}$ Depression predicts adverse health outcomes ${ }^{26}$ and has an impact on mortality, disability, and quality of life. A useful tool for physicians is to question patients about perceived health in order to reveal a hidden depressive state. ${ }^{27}$ Some studies have shown an association between oral health and depression, since it can affect self-esteem and function..$^{28}$ Depression was assessed in separate because it is known to coexist with chronic diseases and to be associated with general functioning. ${ }^{29}$ Interestingly, depression was not associated with bad $\mathrm{SROH}$ in this study.

Frailty is a multifactorial state that also implies vulnerability to adverse outcomes in the elderly. ${ }^{30} \mathrm{In}$ a study with Mexican elderly, the authors reported an increase in the probability of frailty among those who perceived their oral health as worse than others of the same age. ${ }^{31}$ In our study, we found pre-frail older participants to be associated with bad SRGH when compared to frail participants. Interestingly, there was no association between non-frail participants and SRGH and it might have been due to the fact that other variables that might interfere in the relation were not assessed or even because elders did not perceived pre-frailty as a problem since it is a transitional state between being non-frail and being frail. In addition, some of the frail conditions are more severe than others and frailty status includes a higher number of conditions than pre-frailty, which might have influenced the result found. This also may be attributable to other comorbidities that are associated with health perception and may be more frequent in older adults who are not frail.

In this study, literacy, educational level, and having enough money to cover daily expenses represented proxies of socioeconomic status, which may have an effect on access, use of dental care, and preventive information, endorsing positive behavior and attitudes. ${ }^{32}$ High educational level and good socioeconomic condition can affect the level of awareness regarding general and oral health. Individuals with poor conceptual knowledge of oral health issues may not understand and be able to differentiate between good and bad oral health behaviors. ${ }^{33}$ Borrell et al. ${ }^{34}$ observed that less educated and low-income New York adults were more likely to rate their general and oral health as poor, suggesting that socioeconomic characteristics may affect an individual's behaviors and access to resources that could improve feelings of wellbeing and 
life satisfaction. These results are in agreement with ours, in which illiteracy and low educational level were associated with poor perception of general health, and having enough money to live was associated with lower prevalence of bad SROH.

In our study, age and sex did not seem to be related to the two outcomes evaluated. Oksuzyan et al., ${ }^{35}$ who compared population-based self-rated health (SRH) studies conducted in Denmark, Japan, and the US, also observed no substantial differences regarding sex, but found an age-related decline in SRH. In another cross-sectional study, global SRH was found to decline with increasing age. ${ }^{36}$

The hypothesis of reverse causality does not determine whether or not the exposure preceded the outcome; also, temporality cannot be inferred in cross-sectional studies. Furthermore, we only considered self-reports of presence of natural teeth and denture use, which might have affected the results. In addition, we did not assess the quality and functionality of the denture, the number of teeth, or the combination of prosthesis and natural teeth, factors that might contribute to changes in perception. The absence of a dental examination may have lowered the strength of the oral health data beyond the limitation of a self-perceived health assessment. Another limitation of the study is the attrition/response rate for some of the independent variables.

It is important to mention that this study methodology was based on Fried's and Ferrucci's methodology $y^{15,37}$ in which older subjects with low MMSE scores were excluded from the analysis to maintain reliability of self-reported data. The cut-off points for the MMSE were adjusted for the Brazilian population following Brucki et al. ${ }^{13}$ recommendations. According to Ferrucci and collegues, ${ }^{35}$ frailty resulting primarily from reduced cognition was considered a distinct clinical entity even if it was possible to find cognitive decline in frail persons. The authors also concluded that if a certain level of cognitive function is required for the intervention, subjects whose cognitive function is below this level or expected to cross this threshold during the period of the trial should be excluded, highlighting that some abilities required for the study might be questionable for those participants.
In this study, a single question was used for each of the outcomes. A single question has appeal because of the easy interpretation of responses, the low burden on the patient, and the possibility of serving as "screening items". ${ }^{38}$ In addition, the answer to a single question seems to be an integrative summary of one's status in many health-related domains ${ }^{8}$, and its use has been recommended when facing limited resources, sample size restrictions, and patient preferences.$^{38}$ Even though "regular" and "poor" categories were grouped, which could overestimate the prevalence of poor health ${ }^{39}$, the authors considered the categorization adopted the most appropriate for the idea the answers should convey. Besides, it has already been used in previous studies ${ }^{39}$ and it keeps in line with previous research that we published on self-perceived health and selfperceived oral health as outcomes. ${ }^{40}$

Self-rating is an effective and simple option that allows the physician to concentrate on the central problems of patients considering their life situation, determine their needs and expectations, ${ }^{2}$ and employ ancillary personnel to collect information ${ }^{32}$. This method may represent a sensitive, practical, and efficient way to identify groups in which bad SRH could indicate an opportunity for intervention and for monitoring the impact of this intervention. ${ }^{7}$ Furthermore, another FIBRA Study assessing the same volunteers as this study showed that self-reported oral condition is a valid measure for older people, reflecting their clinical oral condition, ${ }^{40}$ therefore SRGH and SROH are both useful in epidemiological studies and primary care.

\section{Conclusion}

Our results suggest that oral health data and income seem to be related to self-perceptions of general and oral health. Therefore, using self-rated questions may allow professionals to gain better understanding of their patients and the aspects important to them that can impact their lives. Epidemiologically, it is a simple way to assess how a population copes.

\section{Acknowledgments}

This research was funded by Fundação de Amparoà Pesquisa do Estado deSão Paulo - Fapesp (2009/51455-2) and Conselho Nacional de Desenvolvimento Científico 
e Tecnológico - CNPq (5550822006/7). Tôrres LHN was supported by Capes. The funders had no role in study design, data collection and analysis, decision to publish, or preparation of the manuscript. The authors have declared that no competing interests exist. Fagundes MLB is supported by Capes.

\section{References}

1. Reddy KK, Reddy BK, Rao AP. Interaction among body composition, self-rated health and functional status of the elderly in an Indian population. Asia Pac J Clin Nutr. 2004;13(1):78-85.

2. Undén AL, Elofsson S. Health from the patient's point of view. How does it relate to the physician's judgement? Fam Pract. 2001 Apr;18(2):174-80. https://doi.org/10.1093/fampra/18.2.174

3. Ruthig JC, Chipperfield JG, Payne BJ. A five-year study of older adults' health incongruence: consistency, functional changes and subsequent survival. Psychol Health. 2011 Nov;26(11):1463-78. https://doi.org/10.1080/08870446.2010.515307

4. Martins AB, Santos CM, Hilgert JB, Marchi RJ, Hugo FN, Padilha DMP. Resilience and self-perceived oral health: a hierarchical approach. J Am Geriatr Soc. 2011 Apr;59(4):725-31. https://doi.org/10.1111/i.1532-5415.2011.03350.x

5. Breidablik HJ, Meland E, Lydersen S. Self-rated health in adolescence: a multifactorial composite. Scand J Public Health. 2008 Jan;36(1):12-20. https://doi.org/10.1177/1403494807085306

6. Menec VH, Chipperfield JG, Perry RP. Self-perceptions of health: a prospective analysis of mortality, control, and health. J Gerontol B Psychol Sci Soc Sci. 1999 Mar;54(2):85-93. https://doi.org/10.1093/geronb/54B.2.P85

7. Perruccio AV, Badley EM, Hogg-Johnson S, Davis AM. Characterizing self-rated health during a period of changing health status. Soc Sci Med. 2010 Nov; $71(9): 1636-43$. https://doi.org/10.1016/j.socscimed.2010.07.042

8. Benyamini $Y$, Leventhal $H$, Leventhal EA. Self-rated oral health as an independent predictor of self-rated general health, self-esteem and life satisfaction. Soc Sci Med. 2004 Sep;59(5):1109-16. https://doi.org/10.1016/i.socscimed.2003.12.021

9. Brennan DS, Teusner DN. Oral health impacts on self-rated general and oral health in a cross-sectional study of working age adults. Community Dent Oral Epidemiol. 2015 Jun;43(3):282-8. https://doi.org/10.1111/cdoe.12152

10. Araújo PF, Silva EF, Silva DD, Sousa ML. Quality of life of adults and elderly individuals who search the Piracicaba Dentistry School service for total dental prostheses. Rev Odontol UNESP. 2008;37(2):109-16.

11. Silva DD, Held RB, Torres SV, Sousa ML, Neri AL, Antunes JL. Self-perceived oral health and associated factors among the elderly in Campinas, Southeastern Brazil, 2008-2009. Rev Saude Publica. 2011 Dec;45(6):1145-53. https://doi.org/10.1590/S0034-89102011000600017

12. Neri AL, Yassuda MS, Araújo LF, Eulálio MC, Cabral BE, Siqueira ME, et al. [Methodology and social, demographic, cognitive, and frailty profiles of community-dwelling elderly from seven Brazilian cities: the FIBRA study]. Cad Saude Publica. 2013 Apr;29(4):778-92. Portuguese. https://doi.org/10.1590/S0102-311X2013000800015

13. Brucki SM, Nitrini R, Caramelli P, Bertolucci PH, Okamoto IH. Sugestões para o uso do mini-exame do estado mental no Brasil. Ara Neuropsiquiatr. 2003 Sep;61 3B:777-81. Portuguese. https://doi.org/10.1590/S0004-282X2003000500014

14. Almeida OP, Almeida SA. [Reliability of the Brazilian version of the ++ abbreviated form of Geriatric Depression Scale (GDS) short form]. Arq Neuropsiquiatr. 1999 Jun;57 2B:421-6. Portuguese. https://doi.org/10.1590/S0004-282X1999000300013

15. Fried LP, Tangen CM, Walston J, Newman AB, Hirsch C, Gottdiener J, et al. Frailty in older adults: evidence for a phenotype. J Gerontol A Biol Sci Med Sci. 2001 Mar;56(3):M146-56. https://doi.org/10.1093/gerona/56.3.M146

16. Okunseri C, Yang M, Gonzalez C, LeMay W, lacopino AM. Hmong adults self-rated oral health: a pilot study. J Immigr Minor Health. 2008 Feb;10(1):81-8. https://doi.org/10.1007/s10903-007-9054-x

17. Matear DW, Locker D, Stephens M, Lawrence HP. Associations between xerostomia and health status indicators in the elderly. J R Soc Promot Health. 2006 Mar;126(2):79-85. https://doi.org/10.1177/1466424006063183

18. De Marchi RJ, Leal AF, Padilha DM, Brondani MA. Vulnerability and the psychosocial aspects of tooth loss in old age: a Southern Brazilian study. J Cross Cult Gerontol. 2012 Sep;27(3):239-58. https://doi.org/10.1007/s10823-012-9170-5

19. Gibson G, Reifenstahl EF, Wehler CJ, Rich SE, Kressin NR, King TB, et al. Dental treatment improves self-rated oral health in homeless veterans-a brief communication. J Public Health Dent. 2008;68(2):111-5. https://doi.org/10.1111/j.1752-7325.2007.00081.x

20. Nyamathi A, Sands H, Pattatucci-Aragón A, Berg J, Leake B, Hahn JE, et al. Perception of health status by homeless US veterans. Fam Community Health. 2004 Jan-Mar;27(1):65-74. https://doi.org/10.1097/00003727-200401000-00007

21. Chen MY. Misperception of Oral Health among Adults in Rural Areas: A Fundamental but Neglected Issue in Primary Healthcare. Int J Environ Res Public Health. 2018 Oct;15(10):2187. https://doi.org/10.3390/ijerph15102187 
Tôrres LHN, Fagundes MLB, Silva DD, Neri AL, Hilgert JB, Hugo FN, et al.

22. Haikal DS, Paula AM, Martins AM, Moreira AN, Ferreira EF. [Self-perception of oral health and impact on quality of life among the elderly: a quantitative-qualitative approach]. Cien Saude Colet. 2011 Jul;16(7):3317-29. Portuguese. https://doi.org/10.1590/S1413-81232011000800031

23. Inukai M, John MT, Igarashi Y, Baba K. Association between perceived chewing ability and oral health-related quality of life in partially dentate patients. Health Qual Life Outcomes. 2010 Oct;8(1):118-23. https://doi.org/10.1186/1477-7525-8-118

24. Kieffer JM, Hoogstraten J. Linking oral health, general health, and quality of life. Eur J Oral Sci. 2008 Oct;116(5):445-50. https://doi.org/10.1111/j.1600-0722.2008.00564.x

25. Milagres CS, Tôrres LH, Neri AL, Sousa MD. [Self-perceived oral health status, chewing ability and longevity in the elderly]. Cien Saude Colet. 2018 May;23(5):1495-506. Portuguese. https://doi.org/10.1590/1413-81232018235.14572016

26. St John PD, Tyas SL, Montgomery PR. Depressive symptoms and frailty. Int J Geriatr Psychiatry. 2013 Jun;28(6):607-14. https://doi.org/10.1002/gps.3866

27. Damian J, Pedro-Cuesta J, Almazán J, Comín-Comín M, Quintanilla MA, Lobo A. Depressive symptoms and associated factors in an older Spanish population positively screened for disability. Int J Geriatr Psychiatry. 2013 Jul;28(7):745-55. https://doi.org/10.1002/gps.3886

28. Hugo FN, Hilgert JB, Sousa MD, Cury JA. Depressive symptoms and untreated dental caries in older independently living South Brazilians. Caries Res. 2012;46(4):376-84. https://doi.org/10.1159/000338382

29. Noël PH, Williams JW Jr, Unützer J, Worchel J, Lee S, Cornell J, et al. Depression and comorbid illness in elderly primary care patients: impact on multiple domains of health status and well-being. Ann Fam Med. 2004 Nov-Dec;2(6):555-62. https://doi.org/10.1370/afm.143

30. Rockwood K. What would make a definition of frailty successful? Age Ageing. 2005 Sep;34(5):432-4. https://doi.org/10.1093/ageing/afil46

31. Castrejón-Pérez RC, Borges-Yáñez SA, Gutiérrez-Robledo LM, Avila-Funes JA. Oral health conditions and frailty in Mexican community-dwelling elderly: a cross sectional analysis. BMC Public Health. 2012 Sep;12(1):773-91. https://doi.org/10.1186/1471-2458-12-773

32. Pattussi MP, Peres KG, Boing AF, Peres MA, da Costa JS. Self-rated oral health and associated factors in Brazilian elders. Community Dent Oral Epidemiol. 2010 Aug;38(4):348-59. https://doi.org/10.1111/j.1600-0528.2010.00542.x

33. Macek MD, Manski MC, Schneiderman MT, Meakin SJ, Haynes D, Wells W, et al. Knowledge of oral health issues among low-income Baltimore adults: a pilot study. J Dent Hyg. 2011;85(1):49-56.

34. Borrell LN, Baquero MC. Self-rated general and oral health in New York City adults: assessing the effect of individual and neighborhood social factors. Community Dent Oral Epidemiol. 2011 Aug;39(4):361-71. https://doi.org/10.1111/j.1600-0528.2010.00603.x

35. Oksuzyan A, Crimmins E, Saito Y, O'Rand A, Vaupel JW, Christensen K. Cross-national comparison of sex differences in health and mortality in Denmark, Japan and the US. Eur J Epidemiol. 2010 Jul;25(7):471-80. https://doi.org/10.1007/s10654-010-9460-6

36. Andersen FK, Christensen K, Frederiksen H. Self-rated health and age: a cross-sectional and longitudinal study of 11,000 Danes aged 45-102. Scand J Public Health. 2007;35(2):164-71. https://doi.org/10.1080/14034940600975674

37. Ferrucci L, Guralnik JM, Studenski S, Fried LP, Cutler GB Jr, Walston JD; Interventions on Frailty Working Group. Designing randomized, controlled trials aimed at preventing or delaying functional decline and disability in frail, older persons: a consensus report. J Am Geriatr Soc. 2004 Apr;52(4):625-34. https://doi.org/10.1111/j.1532-5415.2004.52174.x

38. DeSalvo KB, Fisher WP, Tran K, Bloser N, Merrill W, Peabody J. Assessing measurement properties of two single-item general health measures. Qual Life Res. 2006;15(2):191-201. https://doi.org/10.1007/s11136-005-0887-2

39. Pagotto V, Bachion MM, Silveira EA. [Self-assessment of health by older Brazilians: systematic review of the literature]. Rev Panam Salud Publica. 2013 Apr;33(4):302-10. Portuguese. https://doi.org/10.1590/S1020-49892013000400010

40. Arenas-Márquez MJ, Tôrres LHN, Silva DD, Hilgert JB, Hugo FN, Neri AL, et al. Validity of self-report of oral conditions in older people. Braz J Oral Sci. 2019;18:e191670. https://doi.org/10.20396/bjos.v18i0.8657271 